\title{
MPM Based Simulation for Various Solid Deformation
}

\author{
Yuntao Jiang ${ }^{1}$, Tao Yang ${ }^{1}$, Jian Chang ${ }^{2}$, and Shimin $\mathrm{Hu}^{1}$ \\ 1 Tsinghua University, China, \\ \{jhodinger, yangtao9009\}@gmail.com, \\ shimin@tsinghua.edu.cn \\ 2 Bournemouth University, UK \\ JChang@bournemouth.ac.uk
}

\begin{abstract}
Solid materials are responsible for many interesting phenomena. There are various types of them such as deformable objects and granular materials. In this paper, we present an MPM based framework to simulate the wide range of solid materials. In this framework, solid mechanics is based on the elastoplastic model, where we use von Mises criterion for deformable objects, and the Drucker-Prager model with non-associated plastic flow rules for granular materials. As a result, we can simulate different kinds of deformation of deformable objects and sloping failure for granular materials.
\end{abstract}

Keywords: Solid Simulation, MPM, Elastoplastic Model.

\section{Introduction}

Solid materials exist everywhere in our daily life, and are responsible for many interesting phenomena. Deformable objects, such as chewing gum, toothpaste, and bread dough, undergo elastic and plastic deformation when pressed or stretched. While granular materials, like sand and grain, generate plastic flow and slope failure under large deformation. The numerical simulation of these different materials has been a problem of long standing interest and challenge.

Material Point Method (MPM) is gaining popularity in computer graphics for simulating solid materials, due to its ability to combine the advantage of both Lagrangian and Eulerian approaches. Recently MPM has been successfully applied to simulate granular materials such as sand, and has effectively generated the flow pattern and sloping phenomena [8].

In this work, we show the constitutive models which have been applied in SPH framework also work well in MPM, sometimes even more stable. We use the linear model for elastic deformation, and different yield criterion for the plastic deformation for different materials [1].

In this paper we present an MPM based framework for the wide range of solid materials including deformable objects and granular materials. We introduce a modified version of the Drucker-Prager model with non-associated plastic flow rules for plastic flow of granular materials. The overall framework has a stable 
running performance without the need of extensive tuning, and thus provides a handy tool for complex solid simulation.

\section{Related work}

\subsection{SPH simulation}

SPH shares much with MPM, so here we briefly introduce some SPH based works. SPH was first applied to simulate elastic solid materials by Libersky and Petschek [9]. Gray et al. [6] extended this early work with a method for overcoming the tensile instability that would otherwise lead to numerical fracture. In these works, the strain in deformed solid materials is updated with velocity gradient.

To simulate different materials, different yield criterions are used. Cleary et al. [4] used Von Mises plasticity and linear isotropic hardening to simulate elastoplastic deformation of deformable objects. Bui et al. [3] implemented the Drucker-Prager model with associated and non-associated plastic flow rules to simulate large deformation and post-failure of granular materials, and An et al. [1] extended this work to 3D cases.

In computer graphics, the strain of solid materials is typically computed by comparing the current shape of the solid materials with a reference shape. Müller et al. [10] proposed a particle-based method for elastic, plastic and melting solid materials, using Green-Saint-Venant strain to determine the stress tensor. To approximate the Jacobian of the deformation vector field, a Moving Least Squares approach is employed. Solenthaler et al. [11] use SPH to approximate the Jacobian of the deformation field, which can handle coarsely sampled and coplanar particle configurations. Becker et al. [2] extended their work with a corotational approach to correctly handle rotations. The method using the reference shape can maintain the original shape well, but is unsuitable for applications with extremely large deformations and topological changes.

Yan et al. [15] presented an SPH framework to uniformly handle the interaction between elastoplastic solid and multiple fluids. This framework uses the velocity gradient to update the strain, as in [6] and [1]. Here we follow this method, and extend the solid part with our MPM method.

\subsection{MPM simulation}

Material Point Method(MPM) [14] has been applied to simulate a wide range of solid materials in the past two decades. Stomakin et al. [12] used MPM for simulating snow, and introduced a novel MPM method for heat transport, melting and solidifying materials[13]. Jiang et al. [7] tuned the model in [12] to simulate granular materials. Later Klár et al. [8] introduced the Drucker-Prager plastic flow model into MPM to simulate sand dynamics, and Daviet et al. [5] presented a semi-implicit scheme for granular materials.

Our MPM method is similar to the works mentioned above, but we adopt the constitutive model which has been used in the SPH based work, showing 
that MPM based method is equally flexible and more stable than SPH for these applications.

\section{Solid Mechanics}

The motion of solid materials obeys conservation of mass and conservation of momentum. Since the conservation of mass is naturally preserved by the particle representation, we here only focus on the conservation of momentum

$$
\frac{D \boldsymbol{v}}{D t}=\frac{1}{\rho} \nabla \cdot \boldsymbol{\sigma}+\boldsymbol{g}
$$

where $\boldsymbol{v}$ is the velocity, $\rho$ is the density, $\boldsymbol{g}$ is the gravity, and $\boldsymbol{\sigma}$ is the Cauchy stress tensor determined by the constitutive model of solid materials.

\subsection{Elastic Constitutive Model}

In this section we briefly introduce the elastic constitutive model used by all the solid materials in our framework, and leave the plasticity to section 3.2.

The stress tensor $\boldsymbol{\sigma}$ can be written as

$$
\boldsymbol{\sigma}=-P \boldsymbol{I}+\boldsymbol{s}
$$

where $P$ is the pressure, and $s$ is the deviatoric stress tensor. According to the Hookie's law, the rate of change of $s$ is given by

$$
\frac{D s}{D t}=2 G\left(\dot{\boldsymbol{\epsilon}}-\frac{1}{3} \operatorname{Tr}(\dot{\boldsymbol{\epsilon}}) \boldsymbol{I}\right)
$$

where $G$ is the shear modulus, $\operatorname{Tr}(\cdot)$ is the trace operator, and $\dot{\boldsymbol{\epsilon}}$ is the strain rate tensor, which is given by

$$
\begin{aligned}
\dot{\boldsymbol{\epsilon}} & =\frac{1}{2}\left(\nabla \boldsymbol{v}+\nabla \boldsymbol{v}^{T}\right) \\
\boldsymbol{\omega} & =\frac{1}{2}\left(\nabla \boldsymbol{v}-\nabla \boldsymbol{v}^{T}\right)
\end{aligned}
$$

where $\boldsymbol{\omega}$ is Jaumannn rotation tensor, which is later used to handle rotations. The velocity gradient tensor $\nabla v$ is computed with MPM, which is stated in detail in section 4.

The pressure $P$ can be computed either in SPH scheme or with the constitutive model and is updated with the constitutive model as

$$
\frac{D P}{D t}=-K \operatorname{Tr}(\dot{\boldsymbol{\epsilon}})
$$

where $K$ is the bulk modulus. 
Considering the effect of the rotation, the final equation for updating the stress tensor $\boldsymbol{\sigma}$ is given as

$$
\frac{D \boldsymbol{\sigma}}{D t}=2 G\left(\dot{\boldsymbol{\epsilon}}-\frac{1}{3} \operatorname{Tr}(\dot{\boldsymbol{\epsilon}}) \boldsymbol{I}\right)+K \operatorname{Tr}(\dot{\boldsymbol{\epsilon}}) \boldsymbol{I}+\boldsymbol{\omega} \boldsymbol{\sigma}-\boldsymbol{\sigma} \boldsymbol{\omega}
$$

It is worth mentioning that this approach can handle only small rotations, and approaches like those in [2] or [7] are required to handle larger rotations.

\subsection{Von Mises Plasticity}

When the deformation of deformable objects goes beyond a threshold, the objects are unable to recover their initial shape, and the irreversible part of the deformation is called plastic deformation. The criterion to decide when and how the plastic deformation will take place is called yield criterion.

For deformable objects, the Von Mises criterion is commonly applied:

$$
f\left(J_{2}\right)=J_{2}-Y^{2}=0
$$

where $Y$ is a parameter determining the yield stress, and $J_{2}$ is the second principal invariant of deviatoric stress tensor $\mathbf{s}$, given by: $J_{2}=\frac{1}{2} \mathbf{s}: \mathbf{s}$.

Similar to [15], we assume the solid material has an elastic response at first, calculating a trial stress tensor $\boldsymbol{\sigma}_{t r}$ according to Equation (6), and we can get the trial deviatoric stress tensor by

$$
\boldsymbol{s}_{t r}=\boldsymbol{\sigma}_{t r}-\frac{1}{3} \operatorname{Tr}\left(\boldsymbol{\sigma}_{t r}\right) \boldsymbol{I}
$$

If $f\left(J_{2}\right)>0$, then yield happens, and we update $\mathbf{s}$ as:

$$
s=s_{t r} \sqrt{\frac{Y}{J_{2}}}
$$

Then the stress tensor $\boldsymbol{\sigma}$ is calculated by

$$
\boldsymbol{\sigma}=s+\frac{1}{3} \operatorname{Tr}\left(\sigma_{t r}\right) \boldsymbol{I}
$$

\subsection{Drucker-Prager Model}

For granular materials, we use the Drucker-Prager model with non-associated plastic flow rules here. As in [1], the yield condition $f\left(I_{1}, J_{2}\right)$ and plastic potential function $g\left(I_{1}, J_{2}\right)$ have the following forms, respectively

$$
\begin{aligned}
& f\left(I_{1}, J_{2}\right)=\sqrt{J_{2}}+\alpha_{\phi} I_{1}-k_{c}=0 \\
& g\left(I_{1}, J_{2}\right)=\sqrt{J_{2}}+\alpha_{\psi} I_{1}-C
\end{aligned}
$$

where $I_{1}$ and $J_{2}$ are, respectively, the first and second invariants of the stress tensor $\sigma ; C$ is an arbitrary constant; $\alpha_{\phi}$ and $k_{c}$ are Drucker-Prager's constants, 
which are related to the Coulomb's material constants $c$ (cohesion) and $\phi$ (internal friction); $\alpha_{\psi}$ has the same expression as $\alpha_{\phi}$ and is related to the dilatancy angle $\psi$. These are given as

$$
\begin{aligned}
\alpha_{\phi} & =\frac{\tan \phi}{\sqrt{9+12 \tan ^{2} \phi}} \quad k_{c}=\frac{3 c}{\sqrt{9+12 \tan ^{2} \phi}} \\
\alpha_{\psi} & =\frac{\tan \psi}{\sqrt{9+12 \tan ^{2} \psi}}
\end{aligned}
$$

The stress-strain relationship is given by

$$
\begin{aligned}
\frac{D \boldsymbol{\sigma}}{D t}=\quad & 2 G\left(\dot{\boldsymbol{\epsilon}}-\frac{1}{3} \operatorname{Tr}(\dot{\boldsymbol{\epsilon}}) \boldsymbol{I}\right)+K \dot{\boldsymbol{\epsilon}}+\boldsymbol{\omega} \boldsymbol{\sigma}-\boldsymbol{\sigma} \boldsymbol{\omega} \\
& -\dot{\lambda}\left(3 \alpha_{\psi} K \boldsymbol{I}+\frac{G}{\sqrt{J_{2}}} \boldsymbol{s}\right)
\end{aligned}
$$

where $\dot{\lambda}$ is the rate of change of the plastic multiplier

$$
\dot{\lambda}= \begin{cases}\frac{3 \alpha_{\phi} K \operatorname{Tr}(\dot{\boldsymbol{\epsilon}})+\left(G / \sqrt{J_{2}}\right) s: \dot{\boldsymbol{\epsilon}}}{9 \alpha_{\phi} \alpha_{\psi} K+G} & f\left(I_{1}, J_{2}\right)>0 \\ 0 & f\left(I_{1}, J_{2}\right) \leq 0\end{cases}
$$

\section{Material Point Method}

In MPM particles (material points) are used to track mass, momentum and stress. Specifically, particle $p$ holds position $\boldsymbol{x}_{p}$, velocity $\boldsymbol{v}_{p}$, mass $m_{p}$, and stress $\boldsymbol{\sigma}$. The Lagrangian treatment of these quantities makes the advance step fairly simple. To compute the spatial derivatives of velocity and stress, a regular background Eulerian grid is used.

In each timestep, we first transfer the mass and momentum from particles to the grid, and we compute the velocity gradient at the particles with the grid information. Then we can update the stress on the particles. The forces on grid nodes are computed, and the velocites of the grid nodes are updated. Finally the updated velocity is transferred back to the particles.

\subsection{Interpolation scheme}

To transfer the quantities of particles to the grid, we use a shape function defined as

$$
N_{i}^{h}\left(x_{p}\right)= \begin{cases}\frac{1}{8}\left(1+N_{x} N_{I x}\right)\left(1+N_{y} N_{I y}\right)\left(1+N_{z} N_{I z}\right) & , I \in\left\{N_{p}\right\} \\ 0 & , \text { otherwise }\end{cases}
$$

where $\boldsymbol{N}_{x}=\left(N_{x}, N_{y}, N_{z}\right)$ are the natural coordinates of the evaluation position $\boldsymbol{x}_{p}, \boldsymbol{N}_{I}=\left(N_{I x}, N_{I y}, N_{I z}\right)$ are the natural coordinates of the grid node, and $\left\{N_{p}\right\}$ are the eight nodes of the grid cell containing $\boldsymbol{x}_{p}$. 
The definition of the natural coordinates is given as

$$
\boldsymbol{N}_{x}=\frac{2\left(\boldsymbol{x}_{p}-\boldsymbol{x}_{c}\right)}{h}
$$

where $\boldsymbol{x}_{p}$ is the evaluation position, $h$ is the grid spacing, and $\boldsymbol{x}_{c}$ is the position of the center of the cell where $\boldsymbol{x}_{p}$ lies. Thus the natural coordinate of a position inside the cell ranges from $(-1,-1,-1)$ to $(1,1,1)$.

For more compact notation, we will use $\omega_{i p}=N_{i}^{h}\left(\boldsymbol{x}_{p}\right)$ and $\nabla \omega_{i p}=\nabla N_{i}^{h}\left(\boldsymbol{x}_{p}\right)$. To transfer a scalar $A$ from grid to a particle $p$, we use the shape function as the interpolation function

$$
\begin{aligned}
A_{p} & =\sum_{i} A_{i} \omega_{i p} \\
\nabla A_{p} & =\sum_{i} A_{i} \nabla \omega_{i p}
\end{aligned}
$$

And likewise, to transfer particle data to a grid node $i$, we have

$$
\begin{aligned}
A_{i} & =\sum_{p} A_{p} \omega_{i p} \\
\nabla A_{i} & =-\sum_{i} A_{p} \nabla \omega_{i p}
\end{aligned}
$$

As the node $i$ is shared by eight grid cells, all the particles in these cells contribute to the scalar. The minus sign comes from the fact that $\nabla^{\prime} N_{i}^{h}\left(\boldsymbol{x}_{i}\right)=-\nabla N_{i}^{h}\left(\boldsymbol{x}_{p}\right)$, where $\nabla^{\prime}$ means the derivative operator acts on $\boldsymbol{x}_{i}$.

\section{$4.2 \quad$ Full method}

Here we outline the full update procedure.

1. Rasterize particle data to the grid. The first step is to transfer the mass and momentum from particles to the grid. The mass is transferred using the weighting function $m_{i}^{n}=\sum_{p} m_{p} \omega_{i p}^{n}$. And to conserve momentum, the velocity is transferred as $\boldsymbol{v}_{i}^{n}=\sum_{p} \boldsymbol{v}_{p}^{n} m_{p} \omega_{i p}^{n} / m_{i}^{n}$. The density is transferred as $\rho_{i}^{n}=\frac{m_{i}^{n}}{\sum_{p}\left(m_{p} / \rho_{p}\right) \omega_{i p}^{n}}$.

2. Compute particle velocity gradient. Giving the grid cell that a particle $p$ lies in, the velocity gradient at the particle is computed with the velocities of the cell's eight nodes: $\nabla \boldsymbol{v}_{p}^{n}=\sum_{i} \boldsymbol{v}_{i}^{n}\left(\nabla \omega_{i p}^{n}\right)^{T}$.

3. Update particle stress $\boldsymbol{\sigma}_{p}^{n}$ with the constitutive model in section 3 .

4. Update velocities on grid $\boldsymbol{v}_{i}^{*}$ with $\frac{D v}{D t}=-\frac{1}{\rho_{i}^{n}}\left(\boldsymbol{\sigma}_{p}^{n} \nabla \omega_{i p}^{n}\right)+g$. 


\section{Grid-based body collisions on $\boldsymbol{v}_{i}^{*}$.}

6. Update particle velocities. The new particle velocities are $\boldsymbol{v}_{p}^{n+1}=(1-$ $\alpha) \boldsymbol{v}_{P I C p}^{n+1}+\alpha \boldsymbol{v}_{F L I P p}^{n+1}$, where the PIC part is $\boldsymbol{v}_{P I C p}^{n+1}=\sum_{i} \boldsymbol{v}_{i}^{n+1} \omega_{i p}^{n}$ and the FLIP part is $\boldsymbol{v}_{F L I P p}^{n+1}=\boldsymbol{v}_{p}^{n}+\sum_{i}\left(\boldsymbol{v}_{i}^{n+1}-\boldsymbol{v}_{i}^{n}\right) \omega_{i p}^{n}$. We use $\alpha=0$ for deformable objects and $\alpha=0.05$ for granular materials.

7. Particle-based body collisions on $\boldsymbol{v}_{p}^{n+1}$ with boundaries.

8. Update particle positions using $\boldsymbol{x}_{p}^{n+1}=\boldsymbol{x}_{p}^{n}+\Delta t \boldsymbol{v}_{p}^{n+1}$.

\subsection{Grid-based Collision between different objects}

The most common way of solving the interaction between different objects in MPM is to use multiple grids, one for each object.

We can easily detect the collision of different objects by the overlap of the nodes of different grids, and add collision constraints upon these nodes.

Let's suppose two objects $A$ and $B$ are colliding with each other, and node $i_{A}$ and $i_{B}$ are overlapping with each other. The collision would happen if the following condition is satisfied:

$$
\left(\boldsymbol{v}_{i B}-\boldsymbol{v}_{i A}\right) \nabla m_{i A}<0
$$

where $\boldsymbol{v}_{i A}$ and $\boldsymbol{v}_{i B}$ are the velocity of node $i_{A}$ and $i_{B}$ respectively, and $\nabla m_{i A}$ is the mass gradient of object $A$ defined as $\nabla m_{i A}=\sum_{p} m_{p A} \nabla \omega_{i p A}$, which can be seen as the surface normal of object $A$ pointing outwards.

Hence the collision is handled at the surface of the objects, and only happens when the two object are moving toward each other, and is ignored when they are separating.

Then the velocities at node $i$ is modified as

$$
\begin{aligned}
& \Delta \boldsymbol{v}_{i A}=\left(\boldsymbol{v}_{i B}-\boldsymbol{v}_{i A}\right) \frac{m_{i A} m_{i B}}{m_{i A}+m_{i B}} \\
& \Delta \boldsymbol{v}_{i B}=-\Delta \boldsymbol{v}_{i A}
\end{aligned}
$$

This form of constraint ensures conservation of momentum, and enforces the two nodes to move at the same speed. The penetration is automatically avoided in this way. Although it gives very stable results, it leads to a perfectly inelastic collision, and causes considerable energy lost, so it is not suitable for highly dynamic applications.

\section{Results}

We have simulated several examples to demonstrate the effectiveness of our method, including deformable objects of different plasticity, granular materials, and the interaction between them. 
In Figure 1, a dropping bunny (left), if undergoing pure elastic deformation, bounces up and recover its shape (middle). When plastic deformation happens (right), it can no longer maintain its initial shape and fails to bounce up.
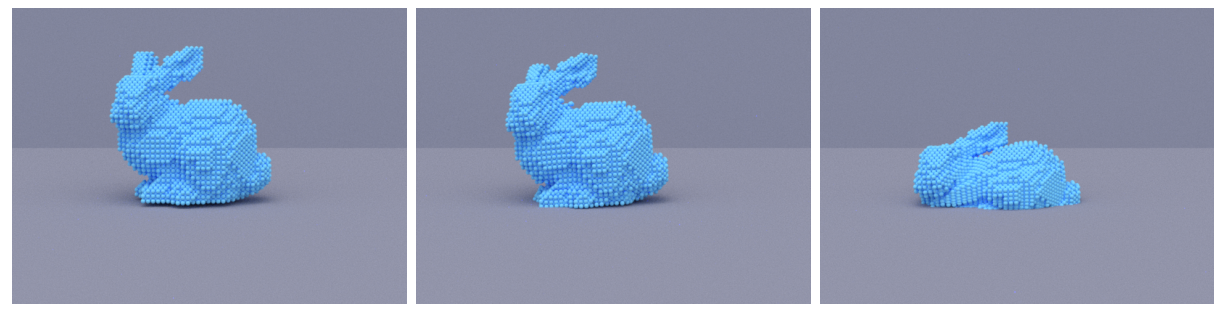

Fig. 1. A bouncing elastic bunny (middle) and a plastic bunny (right).

For granular materials, we set up a notched sand block as one of the cases in [8]. In Figure 2, the block falls down and forms a pile of sand at last. The friction between the ground and the sand is necessary for generating the sloping failure, and we use a friction force proportional to the pressure.
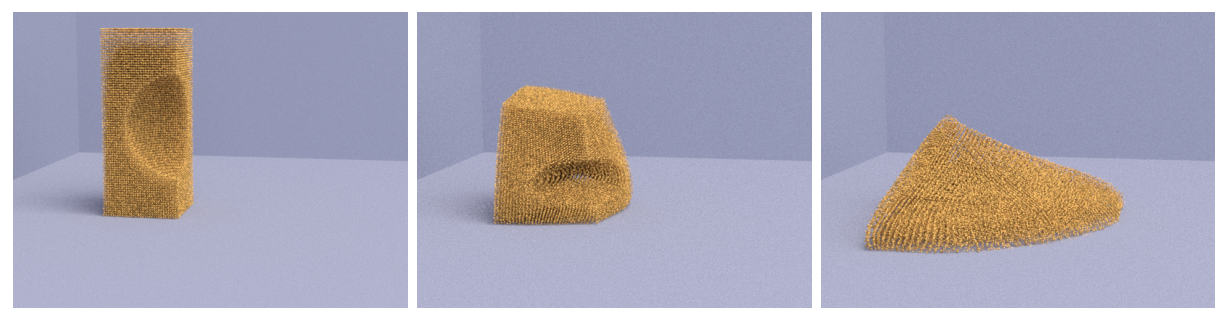

Fig. 2. Sliding and sloping failure of granular materials.

In figure 3 we show a bunny dropping onto a sand ball. The sand ball itself is undergoing deformation, and the bunny hits on it and sinks into the pile of sand in the end.

\section{Discussion and Conclusion}

Comparison with SPH method. MPM is more stable. There is no need to add artificial viscosity to eliminate the tensile and numerical instability. The constitutive models formerly implemented in SPH method can be easily transferred to MPM, and generate good results. However, because the interpolation of velocities from the grid to the particles brings in a loss of information, the phenomena like fragmentations and cracks require different methods to handle, 

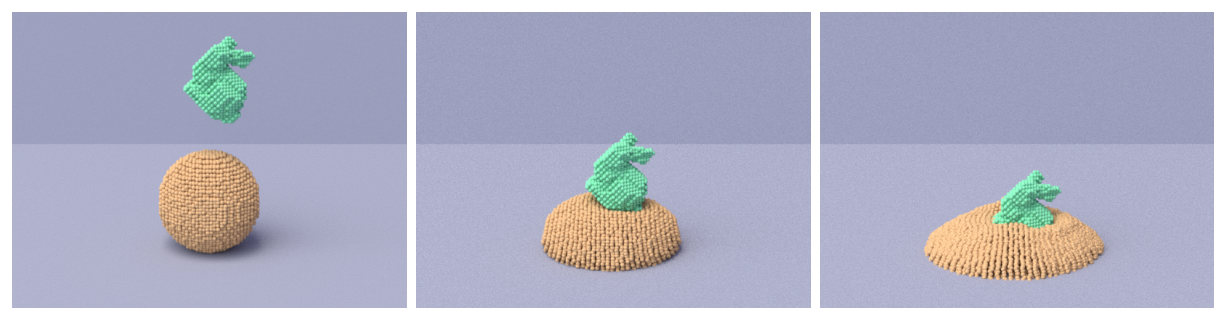

Fig. 3. Interaction between an elastic object and granular materials.

which will be our future work. The APIC method [7] can also be implemented to better perserve the information between the interpolation process, which remains to be tested.

Limitations. The constitutive model we used for solid materials split the strain into two additive part, the elastic strain and the plastic strain. While this is simple and also a good approximation to the exact problem, to truly handle finite deformation, it is better to use a multiplicative approach. Also we would like to investigate more models, hardening, and transitions between different kinds of materials. Besides, the visualization of different materials and their combination requires further study.

Conclusion. We have presented a MPM framework for simulating various solid materials including deformable objects and granular materials. The constitutive models applied in previous SPH based works can be easily adopted to our framework. The collision of different objects are handled in a stable way with the use of the grid.

\section{Acknowledgements}

This work is supported by the People Programme (Marie Curie Actions) of the European Union's Seventh Framework Programme FP7/2007-2013/ under REA grant agreement $\mathrm{n}^{\circ}[612627]$.

\section{References}

1. An, Y., Wu, Q., Shi, C., Liu, Q.: Three-dimensional smoothed-particle hydrodynamics simulation of deformation characteristics in slope failure. Geotechnique 66, 670-680 (Aug 2016)

2. Becker, M., Ihmsen, M., Teschner, M.: Corotated sph for deformable solids. In: Proceedings of the Fifth Eurographics Conference on Natural Phenomena. pp. 27-34. NPH'09, Eurographics Association, Aire-la-Ville, Switzerland, Switzerland (2009), http://dx.doi.org/10.2312EG/DL/conf/EG2009/nph/027-034

3. Bui, H.H., Fukagawa, R., Sako, K., Ohno, S.: Lagrangian meshfree particles method (sph) for large deformation and failure flows of geomaterial using elasticplastic soil constitutive model. International Journal for Numerical and Analytical Methods in Geomechanics 32(12), 1537-1570 (2008), http://dx.doi.org/10.1002/nag.688 
4. Cleary, P.W., Das, R.: The Potential for SPH Modelling of Solid Deformation and Fracture, pp. 287-296. Springer Netherlands, Dordrecht (2008), https://doi.org/10.1007/978-1-4020-9090-5_26

5. Daviet, G., Bertails-Descoubes, F.: A semi-implicit material point method for the continuum simulation of granular materials. ACM Trans. Graph. 35(4), 102:1102:13 (Jul 2016), http://doi.acm.org/10.1145/2897824.2925877

6. Gray, J., Monaghan, J., Swift, R.: Sph elastic dynamics. Computer Methods in Applied Mechanics and Engineering 190(49), 6641 - 6662 (2001), http://www.sciencedirect.com/science/article/pii/S0045782501002547

7. Jiang, C., Schroeder, C., Selle, A., Teran, J., Stomakhin, A.: The affine particle-in-cell method. ACM Trans. Graph. 34(4), 51:1-51:10 (Jul 2015), http://doi.acm.org/10.1145/2766996

8. Klár, G., Gast, T., Pradhana, A., Fu, C., Schroeder, C., Jiang, C., Teran, J.: Drucker-prager elastoplasticity for sand animation. ACM Trans. Graph. 35(4), 103:1-103:12 (Jul 2016), http://doi.acm.org/10.1145/2897824.2925906

9. Libersky, L.D., Petschek, A.G.: Smooth particle hydrodynamics with strength of materials. In: Trease, H.E., Fritts, M.F., Crowley, W.P. (eds.) Advances in the FreeLagrange Method Including Contributions on Adaptive Gridding and the Smooth Particle Hydrodynamics Method. Lecture Notes in Physics, Berlin Springer Verlag, vol. 395, pp. 248-257 (1991)

10. Müller, M., Keiser, R., Nealen, A., Pauly, M., Gross, M., Alexa, M.: Point based animation of elastic, plastic and melting objects. In: Proceedings of the 2004 ACM SIGGRAPH/Eurographics Symposium on Computer Animation. pp. 141151. SCA '04, Eurographics Association, Aire-la-Ville, Switzerland, Switzerland (2004), http://dx.doi.org/10.1145/1028523.1028542

11. Solenthaler, B., Schlfli, J., Pajarola, R.: A unified particle model for fluidsolid interactions. Computer Animation and Virtual Worlds 18(1), 69-82 (2007), http://dx.doi.org/10.1002/cav.162

12. Stomakhin, A., Schroeder, C., Chai, L., Teran, J., Selle, A.: A material point method for snow simulation. ACM Trans. Graph. 32(4), 102:1-102:10 (Jul 2013), http://doi.acm.org/10.1145/2461912.2461948

13. Stomakhin, A., Schroeder, C., Jiang, C., Chai, L., Teran, J., Selle, A.: Augmented mpm for phase-change and varied materials. ACM Trans. Graph. 33(4), 138:1138:11 (Jul 2014), http://doi.acm.org/10.1145/2601097.2601176

14. Sulsky, D., Chen, Z., Schreyer, H.: A particle method for history-dependent materials. Computer Methods in Applied Mechanics and Engineering 118(1), 179 - 196 (1994), http://www.sciencedirect.com/science/article/pii/0045782594901120

15. Yan, X., Jiang, Y.T., Li, C.F., Martin, R.R., Hu, S.M.: Multiphase sph simulation for interactive fluids and solids. ACM Trans. Graph. 35(4), 79:1-79:11 (Jul 2016), http://doi.acm.org/10.1145/2897824.2925897 